\title{
A Novel Indoor Localization Algorithm Design for In-home Healthcare of the Elderly Based on WSN
}

\author{
Wenbo Cheng ${ }^{1,2,3, *}$ and Kai Deng ${ }^{1}$
}

\author{
${ }^{I}$ School of Physics and Electronic Engineering, Yibin University, Ybin, 644000, China; ${ }^{2}$ Institute of Mechanical \\ Engineering, Yibin University, Ybin, 644000, China; ${ }^{3}$ Key Lab of Earth Exploration \& Information Techniques of \\ Ministry of Education, Chengdu University of Technology, Chengdu, 610059, China
}

\begin{abstract}
With the rapid growth of the aging population and the development of pension service industry, positioning service has been played a very important role in healthcare of the elderly. Although many indoor localization algorithms have been developed, there have problems such as low localization precision, high computing complexity and larger memory overhead. The purpose of this work is to construct the system of healthcare based on WSN by using the low power consumption ZigBee communication technology, and present a novel indoor localization algorithm which is called the dictionary database comparison method, after that the algorithm is optimized by using weight factors. Compare of Figs. 6 and 7 shows that the new algorithm has a smaller mean localization error and stronger robustness to indoor environmental disturbance. The results of research and analysis to promote the elderly healthcare will have a positive significance.
\end{abstract}

Keywords: Healthcare of the elderly, localization algorithm, the dictionary database, wireless sensor network.

\section{INTRODUCTION}

In 2014, it is the first time that China's aging population has exceeded 200 million. "Silver China" has been the urgent issue that need to be solved in "The thirteenth national fiveyear plan" [1]. China's aging status as shown in Fig. (1). The fast and irreversible increase of aging population put forward the huge demand for positioning service. The developing level of pension services and industry lags behind, which bring the great opportunities and challenges to healthcare of the elderly.

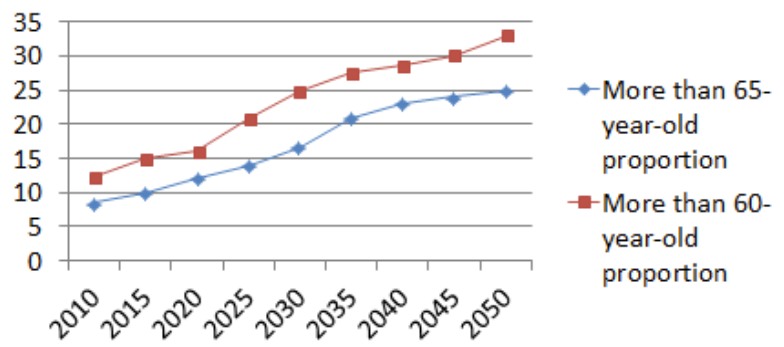

Fig. (1). Growth trend prediction of the chinese aging population proportion (Unit: \%).

Positioning service has been played a very important role in healthcare of the elderly, which has been widely studied by scholars both at home and abroad [2-8]. For example, Sun Shanwu, et al, they presented two methods: one is an improved RSSI-based localization technique in WLAN by using the bounding-box algorithm; the other is an improved binary range search algorithm. The main train of thought is: firstly, pre-rearrange the samples of fingerprint database; secondly, use the linear binary range search algorithm to reduce the number of samples in fingerprint database, finally, compare the real-time localization efficiency [4]. Cheng Wenbo proposed a new method that a wireless healthcare network was constructed by using ZigBee communication technology, and researched the indoor localization precision based on the ZigBee network [5]. Duan Cuicui, et al, they designed a localization system which consists of two parts of the hardware platform and self-developed software platform. They completed the experiments of positioning and tracking based on the two algorithms of symmetrical double-sided two-way ranging and maximum likelihood estimation in this localization system [6]. Guan Weiguo, et al, they proposed an indoor matching localization algorithm based on two-dimensional grid characteristic parameter fusion [7]. The precise terminal location was computed by reference nodes of the matched grid. In order to improve the localization precision, Yang Dongyong, Gu Dongyuan, et al, they presented a conception of Node's RSSI-Similarity Degree [8].

One of the important applications of IEEE802.15.4 in the healthcare of the elderly is monitoring the elderly patient's important data information remotely [9-17]. They can stay their home, but it is important that their physical sign of symptoms can be monitor continuously. The ZigBee mesh networking can be used to collect data form various sensors connected to the elderly patient. The 802.15,4 standard uses 128-bit Advanced Encryption Standard technology to securely transfer data between ZigBee devices and other networks. As shown in Fig. (2). The elderly patient wears a ZigBee device that connects with many sensors, such as the 


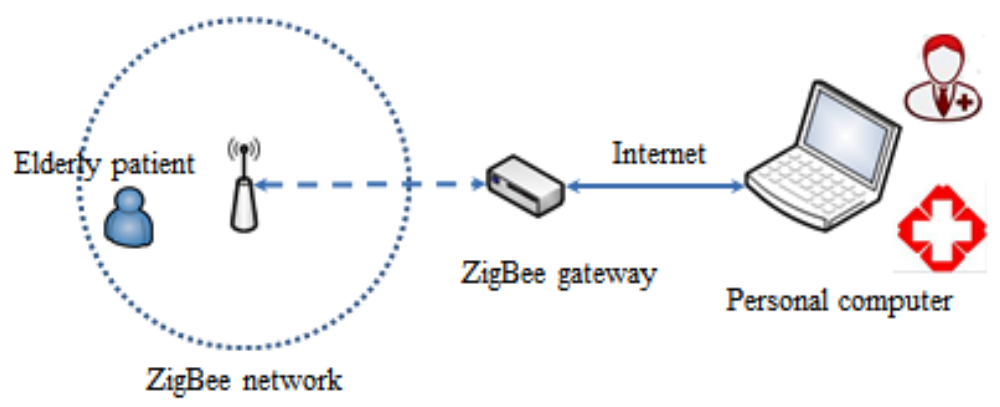

Fig. (2). A simplified diagram of the remote-monitoring system.

Table 1. Parameter specification.

\begin{tabular}{|c|c|c|c|}
\hline Symbol & Description & Symbol & Description \\
\hline \hline$d_{0}$ & Ground proximity the reference distance & $P L(d)$ & Signal strength at distance $d$ \\
\hline$d$ & Ground proximity the reference distance & $X_{\sigma}$ & A log normal random variable of Gaussian distribution \\
\hline$P L\left(d_{0}\right)$ & Signal strength at distance $d_{0}$ & $\alpha$ & Path loss exponent \\
\hline
\end{tabular}

blood pressure sensor, the temperature sensor, the heart rate sensor and so on. They collect the vital information on a periodic basis. Then these mass of physical data are transmitted to a ZigBee gateway which provides the interface between a ZigBee mesh networking and other networks [13, 14]. For example, the elderly patient's physical sign of symptoms data can be transmitted over the internet protocol network to a personal computer that the physician or nurse uses to monitor the elderly patient. The remote-monitoring system can help physician or nurse improve the elderly patient care and provide dietary advice.

If location data is difficult to obtain or the positioning accuracy is very low, then the meaningless information will make the remote-monitoring system unnecessary. As mentioned above, although many indoor location algorithms have developed, but there are still problems such as low precision, high computing complexity and larger the memory overhead. In order to solve these problems, the paper tries to use low power consumption ZigBee communication technology and to construct the wireless network of healthcare based on WSN, and propose a novel indoor localization algorithm which is called the dictionary database comparison method, after that the method is further optimized.

\section{RESEARCH METHOD}

RSSI-based localization technique is the technology which uses the measuring signal attenuation degree to obtain the actual distance between the two nodes from the transmitter to the receiver. The shadowing model [14] is defined by

$$
P L(d)=P L\left(d_{0}\right)+10 \alpha \lg \left(d / d_{0}\right)+X_{\sigma}
$$

This model consists of two parts. Table 1 gives the parameters list, where alpha is the path loss exponent and is usually determined empirically by field measurements. $P L\left(d_{0}\right)$ can be calculated by using the free space equation.
The first parts predict the received power $P L(d)$ at distance d relative to the power at an arbitrarily close-in distance $d_{0}$ (e.g. 1-metre). The second part of the shadowing model reflects the variation of the received signal power at a certain distance and is a log normal random variable of Gaussian distribution designated by $X_{\sigma}$. If we do not consider the effect of $X_{\sigma}$, the actual distance d between two nodes is given by

$d=d_{0} * \exp \left(\left(P L(d)-P L\left(d_{0}\right)\right) / 10 \alpha\right)$

In this system, $R S S I_{\text {mod }}\left(d_{0}\right)$ and $R S S I_{\text {mod }}\left(d_{i}\right)$ represents the theoretical value of the signal strength at distance $d_{0}$ and $d_{i}$ which is a distance with the $i$ th node, $R S S I_{\text {mea }}\left(d_{i}\right)$ represents the actual value of the signal strength at distance $d_{i}$, so root-mean-squared error can be described by

$F(\alpha)=\sqrt{\frac{1}{N} \sum_{i=1}^{N}\left[R S S I_{\text {mea }}\left(d_{i}\right)-R S S I_{\text {mod }}\left(d_{i}\right)\right]^{2}}$

It is easy to derive the equation (4) by the equation (1).

$R S S I_{\text {mod }}\left(d_{i}\right)=R S S I_{\text {mod }}\left(d_{0}\right)-10 \alpha \lg \left(d_{i} / d_{0}\right)$

While

$\partial F(\alpha) / \partial \alpha=0$

We can easily obtain the optimal solution of the path loss exponent.

\section{RESULTS AND ANALYSIS}

\subsection{Algorithm Design}

Triangulation location method can obtain the position of the unknown nodes which is intersection point of circles, the center of circle is location of three or more of the anchor 


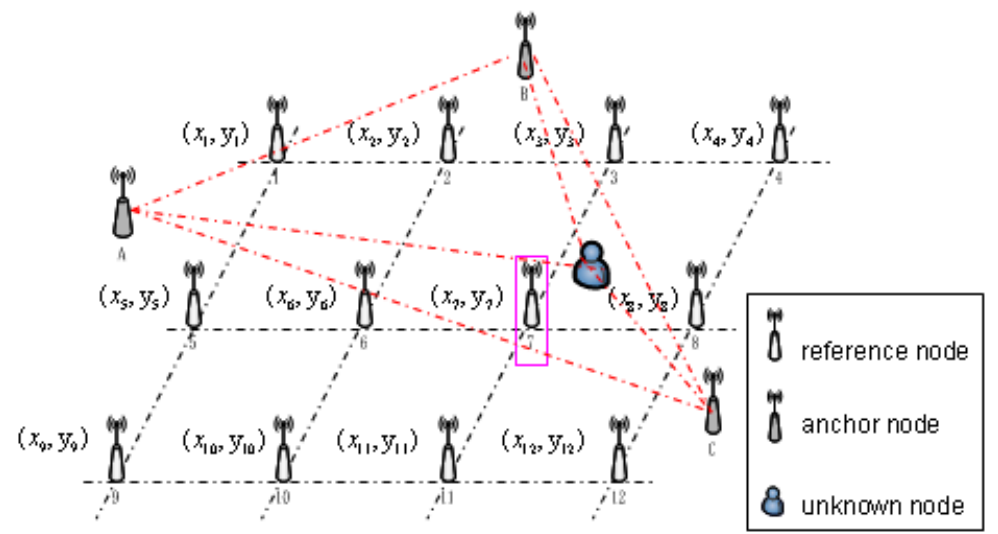

Fig. (3). Illustration of the dictionary database comparison method. Purple box indicates the localization result of comparing with the dictionary database.

Table 2. Parameter specification.

\begin{tabular}{|c|c|c|c|}
\hline Symbol & Description & Symbol & Description \\
\hline \hline$N$ & The anchor node number & \multirow{2}{*}{$D_{j}(i)$} & $\begin{array}{c}\text { Signal strength between } j \text { th referenced node of the } \\
\text { dictionary library and } i \text { th anchor node }\end{array}$ \\
\hline$\beta$ & Distance eigenvalue & T he minimum signal strength differences \\
\hline$P L(i)$ & Signal strength between unknown node and $i$ th anchor node & $d_{d-V}$ & \\
\hline
\end{tabular}

nodes, and the radius is the measured distance between anchor nodes and unknown nodes. This method has the advantages of simple operation and easy to implement. However, they do not meet in a bit and even produce singular matrix, location information is uncertain [18]. In order to improve this situation, and try to reduce the localization error, the paper proposes a novel indoor localization algorithm based on the dictionary database comparison method.

Specific operation steps of the algorithm are described as follows:

Step 1: In the monitoring area, deploy the anchor nodes and the reference nodes.

Step 2: Measure signal strength between the reference nodes and anchor nodes, and then establish the dictionary library of the reference nodes position itself and measurement of the location data.

Step 3: Remove all the reference node, and then measure signal strength between the unknown nodes and multiple the anchor nodes.

Step 4: Compare with the dictionary database location data, and then choose the minimize differences compared with the reference node's position as the coordinates of the unknown node.

As shown in Fig. (3), it includes 12 reference nodes. Their coordinates and each signal strength measurement with the anchor nodes $\mathrm{A}, \mathrm{B}$ and $\mathrm{C}$ are recorded in the dictionary database. When an unknown node moves into the monitoring area, we use the equation (6) to calculate the minimum signal strength differences by comparing the actual measured value with the dictionary database. Table 2 gives the parameters list.

$$
d_{d-V}=\frac{1}{N}\left(\sum_{i=1}^{N}\left|P(i)-D_{j}(i)\right|^{\beta}\right)^{1 / \beta}
$$

The positioning accuracy of this method is affected by the number of reference nodes. In the same monitoring area, the more we deploy the reference nodes, the higher the positioning accuracy. But the dictionary database will occupy more memory resources, and consume the more time. In order to use limited information in the dictionary database and improve the positioning accuracy, we can optimize the position coordinates of the unknown nodes. As shown in Fig. (4).

Firstly, we find out the $m$ number of the smaller $d_{d-V}$ by the equation (6). Secondly, we use inverse of $d_{d-V}$ as a weight factor, and can optimize the coordinates of the unknown nodes. The coordinates are denoted by:

$$
(x, y)=\sum_{i=1}^{m} \frac{\left(x_{i}, y_{i}\right)}{\left(d_{d-V_{i}}+d_{d-V_{0}}\right)} / \sum_{i=1}^{m} \frac{1}{\left(d_{d-V_{i}}+d_{d-V_{0}}\right)}
$$

where $\left(x_{i}, y_{i}\right)$ represents the coordinates of the $i$ th reference node in the $m$ number of the smaller $d_{d-V}, d_{d-V_{i}}$ represents the signal strength differences between the unknown node and the $i$ th reference node in the dictionary database, $d_{d-V_{0}}$ represents the minimum in order to avoid division cause singular matrix.

\subsection{ZigBee Networks Design}

IEEE802.15.4 standard was developed due to the unsuitability of current wireless standard for low data rate personal area networks. It is a simple packet data protocol 


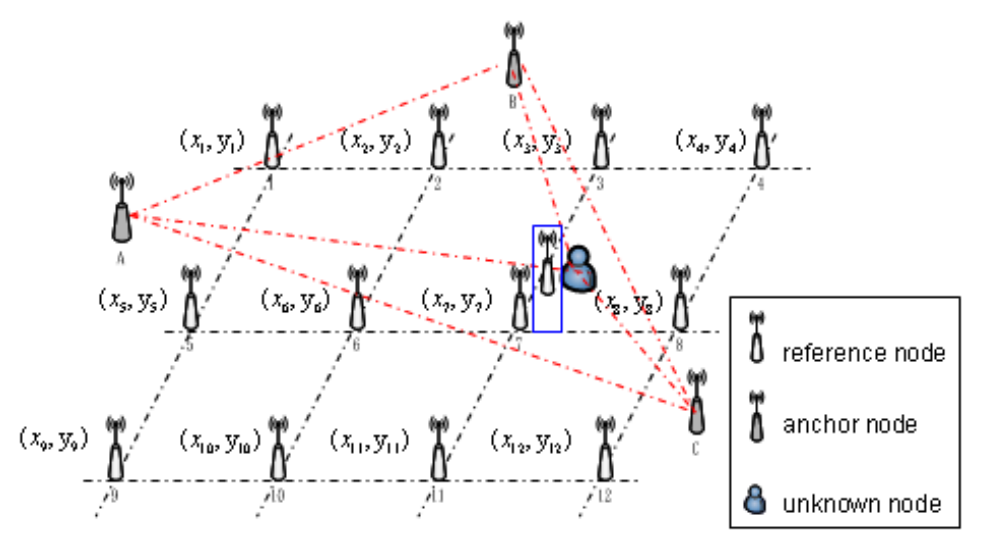

Fig. (4). The optimized illustration of the dictionary database comparison method. Blue box indicates the localization result of the introduction of weight.

for light-weight wireless network and specifies the media access control (MAC) and physical (PHY) networking layers [19]. PHY in this standard is designed to operate in the $2.4 \mathrm{GHz}$ industrial, scientific, and medical (ISM) the frequency band. PHY is based on direct sequence spread spectrum (DSSS) methods that result in low-cost digital integrated circuit implementation, and share the basic packet structure for low duty cycle, low-power operation. In this research, the $2.4 \mathrm{GHz}$ PHY has been chosen due to the world wide availability and the wider availability of wireless transceivers at this frequency.

ZigBee is a wireless network standard which is based on the PHY and MAC layers of IEEE 802.15.4 standard. ZigBee technology takes full advantage of it and adds the logical network, security, and application software. ZigBee involves applications requiring low-power-consumption, low-cost and low-data-rates as well as advanced features such as mesh network and security [20-22]. In this research, the mesh network topology has been chosen due to two reasons: on the one hand, routers are able to communicate directly with each other and forming multiple routes, on the other hand, the topology has more efficient message propagation. As shown in Fig. (5).

The CC2430 is a true System-on-Chip solution for ZigBee and combines the excellent performance of the RF transceiver with an industry-standard enhanced $8051 \mathrm{MCU}$, $32 / 64 / 128$ kByte flash, 8kB of RAM and many other powerful features [23]. Combined with TI's leading ZigBee protocol stack, it is the market's most competitive ZigBee solution. ZigBee transceiver chip CC2430 was chosen for the anchor nodes which has a static location. These nodes must be configured with $\mathrm{X}$ and $\mathrm{Y}$ value that correspond to the physical location. The main task is to provide a "reference" packet that contains X and Y coordinates to the unknown node, also referred to as an anchor node. Since these nodes are not using the hardware location engine at all, so we can choose the chip CC2430 for the purpose.

CC2431 was chosen for the unknown nodes and reference nodes. An unknown node will communicate with the closest anchor nodes, collecting X, Y and RSSI for each of these nodes, and calculate its position based on the parameter input using the location engine hardware. Afterwards the calculated position should be sent to a control station which could be a PC or another node in the system.

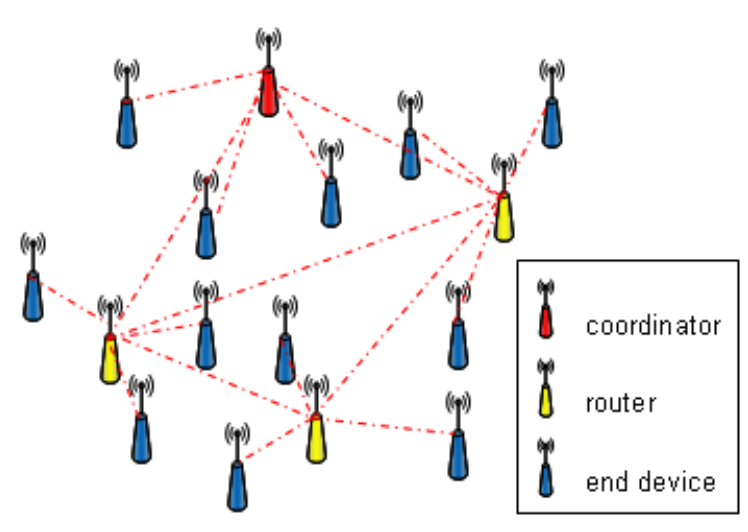

Fig. (5). ZigBee mesh topology.

The CC2431 with location engine is a true system-onchip for ZigBee/IEEE 802.15.4 wireless sensor networking solution, so it will be natural to use the location engine in a ZigBee mesh network. The location algorithm used in the CC2431 Location Engine [23] is based on Received Signal Strength Indicator values. The main feature of the location engine is that the location calculation can be performed at each unknown node, hence the algorithm is decentralized. This property reduces the amount of data transferred in the mesh network, since only the calculated position is transferred, not the data used to perform the calculation.

Table 3 shows all necessary input to the location hardware. The following is a brief introduction.

Table 4 shows output to the location hardware. The following is a brief introduction.

When the chip CC2430/31 receives a packet it will automatically add an RSSI value to the received packet. The RSSI value is always averaged over the 8 first symbol periods $(128 \mu)$ [23]. This RSSI value is represented as a one byte value, as a signed 2's complement value. When a packet is read from the FIFO on the chip CC2431 the second last byte will contain the RSSI value that was measured after receiving 8 symbols of the actual packet. Even if the RSSI value is captured at the same time as the data packet is received, the RSSI value will reflect the intensity of received signal strength at that time, not necessarily the signal power belonging to the received data. This gives the opportunity for the RSSI value to be erroneous when a large number of 
Table 3. Hardware inputs parameters.

\begin{tabular}{|c|c|c|c|}
\hline Name & Min.value & Max.value & Description \\
\hline \hline A & 30 & 50 & The absolute RSSI value in dBm one meter apart for a transmitter. \\
\hline N_index & 0 & 31 & This value represent the signal propagation exponent, this value depends on the environment. \\
\hline RSSI & 40 & 95 & Received Signal Strength Indicator this value is measured in dBm. The location engine using the absolute \\
value as input.
\end{tabular}

Table 4. Location engine output.

\begin{tabular}{|c|c|c|c|}
\hline Name & Min.value & Max.value & Description \\
\hline \hline $\mathrm{X}, \mathrm{Y}$ & 0 & 63.75 & $\begin{array}{r}\text { These values represent the calculated } \mathrm{X} \text { and } \mathrm{Y} \text { coordinates relatively to a } \\
\text { fixed point. The values are in meters. }\end{array}$ \\
\hline
\end{tabular}

Table 5. Received data packet.

\begin{tabular}{|l|l|l|l|l|l|l|l|}
\hline $\mathrm{n}$ & $\mathrm{D}(0)$ & $\mathrm{D}(1)$ & $\mathrm{D}(2)$ & $\ldots$ & $\mathrm{D}(\mathrm{n}-2)$ & $\mathrm{RSSI}$ & $\mathrm{CRC}$ \\
\hline
\end{tabular}

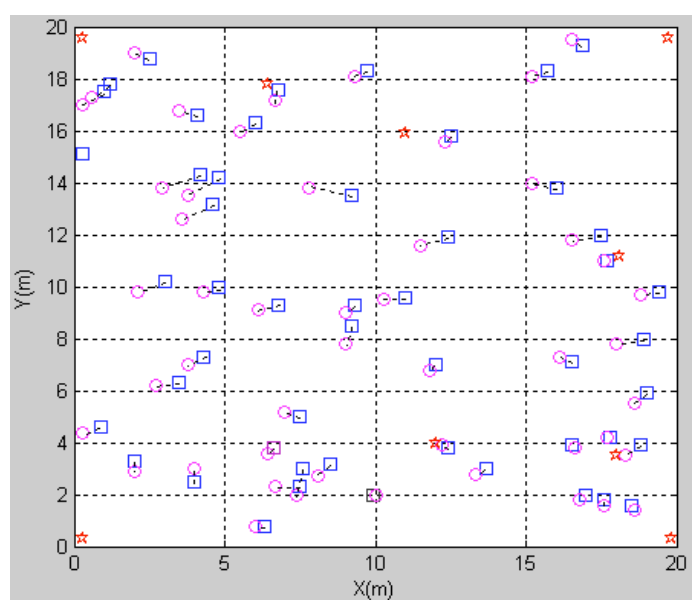

(a)

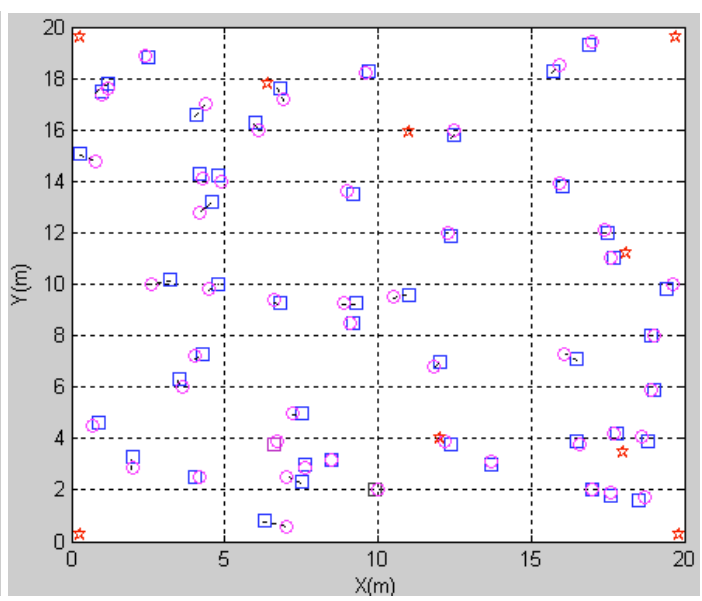

(b)

Fig. (6). a) The localization results before optimization. b) After optimization.

nodes are talking on the same channel at the same time as the RSSI value is captured [23].

The chip CC2430/31 contains a register termed RSSI. This register holds the same values as described in Table 5, but it is not locked when a packet is received, hence the register value should not be used for further calculations. Only the locked RSSI value attached to the received data can be interpreted as the RSSI value measured exactly when the data is received [23].

\section{CONCLUSION}

In order to test the maneuverability of the wireless network and effectiveness of the new algorithm, we assume the network is a $20 \times 20 \mathrm{~m}^{2}$ square area. There are four anchor nodes which are placed at the four corners, the rest of five anchor nodes (the red stars) randomly distributed within the network, the number of unknown nodes (the blue squares) is 50 , all positioning coordinates are marked as the purple circle.

As for the localization accuracy, we compute the mean localization error using the RMSE given by the equation (3) in MATLAB 2007 on a Pentium(R) Dual-Core CPU. When the end device under test has associated with the coordinator and begun sending packets the data acquisition was started by running the MATLAB script from computer and the position data was recorded.

As shown in Figs. (6a) and (6b), where the communication range is $R_{C}=10 \mathrm{~m}, \alpha=2$ and $X_{\sigma}=10.7$. We can easily observe that in Fig. (6a), a few nodes get 
flipped solutions, therefore, it will result in a larger localization error of $R M S E=0.65 \mathrm{~m}$, while Fig. (6b) has the smallest localization error of $R M S E=0.32 \mathrm{~m}$, due to the optimization of algorithm.

\section{CONFLICT OF INTEREST}

The authors confirm that this article content has no conflict of interest.

\section{ACKNOWLEDGEMENTS}

A Project Supported by Scientific Research Fund of Sichuan Provincial Education Department (Grant No.14ZA0269), Scientific Research Key Project of Yibin University (Grant No. 2013QD02) and the National Natural Science Foundation of China (Grant No. 61201266).

\section{REFERENCES}

[1] Population ageing information on China National Committee on Ageing. http://en.cncaprc.gov.cn/, 2014.

[2] R. Gururajan, S. Murugesan, and J. Soar, "Introducing mobile technologies in support of healthcare", Cutter IT Journal: the Journal of Information Technology Management, pp. 12-18, 2005.

[3] M. Karla, and N. Felix, "A Heterogeneous network management approach to wireless sensor networks in personal healthcare environments", Faculty of Science University of Technology, Sydney, 2008.

[4] S. W. Sun, N. Wang, J. Chen, "Improved RSSI-based localization method using bounding-box algorithm in WLAN", Computer Science, pp. 99-103, 2014

[5] W. B. Cheng, "Construction of WSN and application of localization for in-home healthcare of the elderly", International Conference on Information Technology and Management Innovation, pp. 943-947, 2012.

[6] C. C. Duan, R. R. Wang, J. Z. Wang, "Localization system for staff in high-risk production area based on wireless sensor networks", Chinese Journal of Sensors and Actuators, pp. 1599-1602, 2012.

[7] W. G. Guan, B. C. Lu, "Indoor matching localization algorithm based on two-dimensional grid characteristic parameter fusion", Journal of Computer Applications, pp. 2464-2467, 2471, 2014.

[8] D. Y. Yang, D. Y. Gu, X. J. Fu, "An indoor location algorithm based on RSSI-Similarity degree", Chinese Journal of Sensors and Actuators, pp. 264-268, 2009.

[9] H. Seok, K., S. Joo-Han, and L. Seok, "Energy-Efficient Traffic Scheduling in IEEE 802.15.4 for Home Automation Networks", Consumer Electronics, IEEE Transactions on, pp. 369-374, 2007.
[10] C. Li, H.B. Li, and R. Kohno, "Performance evaluation of IEEE 802.15.4 for wireless body area network", The Proc. of IEEE International Conference on ICC Workshops, pp. 1-5, 2009.

[11] N. F. Timmons, and W. G. Scanlon, "Analysis of the performance of IEEE 802.15.4 for medical sensor body area networking", The Proc. of IEEE SECON, pp. 4-7, 2004.

[12] H. Li, and J. Tan, "An ultra-low-power medium access control protocol for body sensor network", $27^{\text {th }}$ Annua International Conference of the Engineering in Medicine and Biology Society 2005, pp. 2451-2454, 2005.

[13] A. Milenkovic, C. Otto, and E. Jovanov, "Wireless sensor network for personal health monitoring: Issues and an implementation", Comput. Commun, pp. 2521-2533, 2006.

[14] J. M. Choi, B. O. Kim, B. S. Hwang, "A system for ubiquitous health monitoring in the bedroom via a Bluetooth network and wireless LAN”, Conf Proc IEEE Eng Med Biol Soc, pp. 3362-3365, 2004.

[15] Z. Chen, H. Chao, L. Jingsheng, and L. Shoubin, "Protocol architecture for wireless body area network based on nRF24L01", The Proc. of IEEE International Conference on Automation and Logistics 2008, pp. 3050-3054, 2008

[16] G. Fang, and E. Dutkiewicz, "Body MAC: Energy efficient TDMA-based MAC protocol for wireless body area networks", The Proc. of International Symposium on Communications and Information Technologies 2009. Incheon, Korea, 2009.

[17] H. Su, and X. Zhang, "Battery-dynamics driven TDMA. MAC protocols for wireless body-area monitoring networks in healthcare applications", IEEE J. Sel. Areas Commun, pp. 424-434, 2009.

[18] W. B. Cheng, H. J. Wang, "The key techniques of mine personnel localization system based on wireless sensor network", Microelectronics \& Computer, p. 165-168, 2012.

[19] IEEE Std.802.15.4: "Wireless medium access control (MAC) and physical layer (PHY) specifications for low data rate wireless personal area networks (WPAN)", IEEE: Piscataway, NJ, USA, 2006.

[20] E. Callaway, P. Gorday, and L. Hester, "Home networking with IEEE 802.15.4: a developing standard for low-rate wireless personal area networks, IEEE Communication Magazine, pp. 70-77, 2002.

[21] B. Bougard, F. Catthoor, D. C. Daly, A. Chandrakasan, and W. Dehaene, "Energy efficiency of the IEEE 802.15.4 standard in dense wireless microsensor networks: modeling and improvement perspectives", Proceedings of Design (2005), Automation and Test in Europe, Munich, Germany, 7-11 March 2005, pp. 196-201, 2005

[22] A. Gaur, A. Prakash, S. Joshi, and D. P. Agrawal, "Polynomial based scheme (PBS) for establishing Authentic Associations in Wireless Mesh Networks", Journal of Parallel and Distributed Computing, pp. 338-343, 2010.

[23] K. Aamodt, "CC2431 location engine, Application Note AN042", Texas Instruments Incorporated: Texas, pp. 4-6, 2010.

(C) Cheng and Deng; Licensee Bentham Open.

This is an open access article licensed under the terms of the (https://creativecommons.org/licenses/by/4.0/legalcode), which permits unrestricted, noncommercial use, distribution and reproduction in any medium, provided the work is properly cited. 